\title{
Leading and higher twists in proton, neutron and deuteron unpolarized structure functions $F_{2}$
}

\section{S. Simula ${ }^{\mathrm{a}}$}

Istituto Nazionale di Fisica Nucleare, Sezione di Roma 3, Via della Vasca Navale 84, I-00146 Roma, Italy

Received: 23 November 2006

Published online: 9 March 2007 - (C) Società Italiana di Fisica / Springer-Verlag 2007

\begin{abstract}
We summarize the results of a recent global analysis of proton and deuteron $F_{2}$ structure function world data performed over a large range of kinematics, including recent measurements done at JLab with the CLAS detector. From these data the lowest moments $(n \leq 10)$ of the unpolarized structure functions are determined with good statistics and systematics. The $Q^{2}$ evolution of the extracted moments is analyzed in terms of an OPE-based twist expansion, taking into account soft-gluon effects at large $x$. A clean separation among the leading- and higher-twist terms is achieved. By combining proton and deuteron measurements the lowest moments of the neutron $F_{2}$ structure function are determined and its leading-twist term is extracted. Particular attention is paid to nuclear effects in the deuteron, which become increasingly important for the higher moments. Our results for the non-singlet, isovector $(p$ - $n)$ combination of the leading-twist moments are used to test recent lattice simulations. We also determine the lowest few moments of the higher-twist contributions, and find these to be approximately isospin independent, suggesting the possible dominance of $u d$ correlations over $u u$ and $d d$ in the nucleon.
\end{abstract}

PACS. 13.60.Hb Total and inclusive cross sections (including deep-inelastic processes) - 12.38.Cy Summation of perturbation theory - 12.38.Qk Experimental tests - 12.38.Lg Other nonperturbative calculations

In the past few years, thanks to the performance of the CLAS detector of the Hall $\mathrm{B}$ at Jefferson lab, the unpolarized structure functions $F_{2}\left(x, Q^{2}\right)$ of both proton and deuteron have been measured precisely in a wide continuous interval of values of the Bjorken variable $x$ and of the squared four-momentum transfer $Q^{2}[1,2]$. In particular, the $F_{2}$ structure functions have been extracted over the whole resonance region $(W \leq 2.5 \mathrm{GeV})$ below $Q^{2} \simeq 4.5(\mathrm{GeV} / c)^{2}$. These measurements, together with existing world data, have allowed the determination of the moments $M_{n}\left(Q^{2}\right)$ of the structure function $F_{2}$ up to $n=10$, drastically reducing the uncertainties related to data interpolation and extrapolation as well as providing the most accurate evaluation of the $Q^{2}$-dependence of the moments starting from $Q^{2} \simeq 0.1(0.5)(\mathrm{GeV} / c)^{2}$ for proton (deuteron) up to $Q^{2} \simeq 100(\mathrm{GeV} / c)^{2}$.

A very powerful tool for analyzing in $\mathrm{QCD}$ the $Q^{2}$ dependence of the moments $M_{n}\left(Q^{2}\right)$ is represented by the Operator Product Expansion (OPE). Through OPE the moments are given as a series expansion in terms of matrix elements of local operators, namely

$$
M_{n}\left(Q^{2}\right)=\sum_{\tau=2}^{\infty} E_{n \tau}\left(\mu, Q^{2}\right) O_{n \tau}(\mu)\left(\frac{1}{Q^{2}}\right)^{\frac{1}{2}(\tau-2)},
$$

a e-mail: simula@roma3.infn.it where $\mu$ is the renormalization scale, $O_{n \tau}(\mu)$ is the reduced matrix element of local operators with definite spin $n$ and twist $\tau$, related to the non-perturbative structure of the target, and $E_{n \tau}\left(\mu, Q^{2}\right)$ is a short-distance coefficient calculable in $\mathrm{pQCD}$ thanks to the asymptotic freedom.

The first term of the series (1) corresponds to the Leading Twist (LT) and is determined by single-parton distributions in the target. Subsequent terms are the so-called Higher Twists (HTs), which depend on the interactions among partons (i.e., on multi-parton correlations). Their determination is considerably more challenging both experimentally and theoretically.

In refs. [1] and [2] both the LT and the HTs (up to twist-6) have been extracted from the proton and deuteron moments, respectively. Two important features of our twist analysis should be mentioned: 1) the analyzed moments are Nachtmann moments, and not Cornwall-Norton ones; 2) the perturbative evolution of the LT is calculated using soft-gluon resummation techniques.

We have adopted the Nachtmann definition of the moments in order to get rid of the target-mass corrections, which are power corrections of kinematical origin and affect the Cornwall-Norton (CN) definition of the moments. The use of Nachtmann moments allows to extract in a clean way dynamical HTs, which are the only ones related to multi-parton correlations. The target-mass corrections 


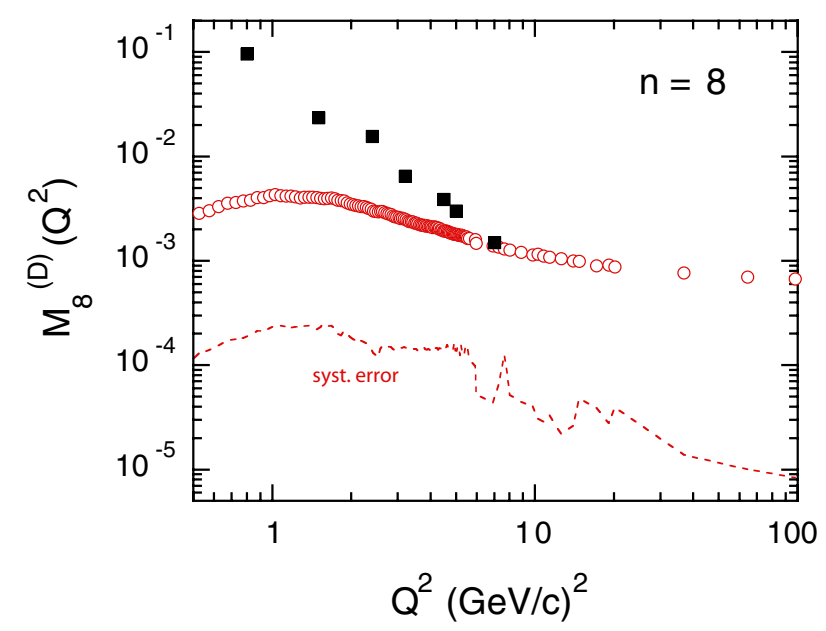

Fig. 1. Moment $M_{8}^{(D)}\left(Q^{2}\right)$ of the deuteron versus $Q^{2}$. Open dots are the results of ref. [2] using the Nachtmann definition of the moments, while full squares correspond to the $\mathrm{CN}$ moment as determined in ref. [3]. The reported systematic errors have been precisely estimated only in ref. [2].

can be subtracted in principle from the $\mathrm{CN}$ moments, but this procedure requires a precise knowledge of the LT moments at large $n$. Moreover, the contribution of targetmass corrections is sizable just in the region of $Q^{2} \simeq$ few $(\mathrm{GeV} / c)^{2}$, where HTs are expected to show up. This point is clearly illustrated in fig. 1, where the Nachtmann moment $M_{8}^{(D)}\left(Q^{2}\right)$ is compared with the corresponding $\mathrm{CN}$ one as determined in ref. [3]. It is also worthwhile to note the much larger number of very accurate data points obtained thanks to the CLAS detector.

As for the perturbative evolution of the LT, the presence of large logarithms in the coefficient function at large $n$ requires to go beyond any fixed-order approximation. As shown in ref. [4] for a reliable extraction of both LT and HTs it is crucial to apply the resummation of soft gluons. Moreover, for internal consistency the value of $\alpha_{s}\left(M_{Z}^{2}\right)$ has been extracted in ref. [5] by analyzing the large- $Q^{2}$ behavior of the proton moments of ref. [1] at the next-to-leading logarithmic accuracy used for the soft-gluon resummation.

The extracted LT components of proton and deuteron moments can be combined to form the LT moments of the neutron $F_{2}$ structure function. In the impulse approximation the deuteron structure function can be written as the sum of two terms: $F_{2}^{(D)}=F_{2}^{(D, c o n v .)}+F_{2}^{(D, o f f)}$, where the first term is given by a convolution of the nucleon structure function and the nucleon momentum distribution function in the deuteron, $f^{(D)}$, while the second term represents nuclear off-shell plus relativistic corrections ${ }^{1}$. Thus, the neutron moments can be obtained from

$$
M_{n}^{(n)}\left(Q^{2}\right)=2 M_{n}^{(D)}\left(Q^{2}\right) \frac{1-\Delta_{n}^{(o f f)}}{N_{n}^{(D)}}-M_{n}^{(p)}\left(Q^{2}\right)
$$

\footnotetext{
${ }^{1}$ For the moment with $n=2$ shadowing and meson exchange current should be also considered (see ref. [6]).
}

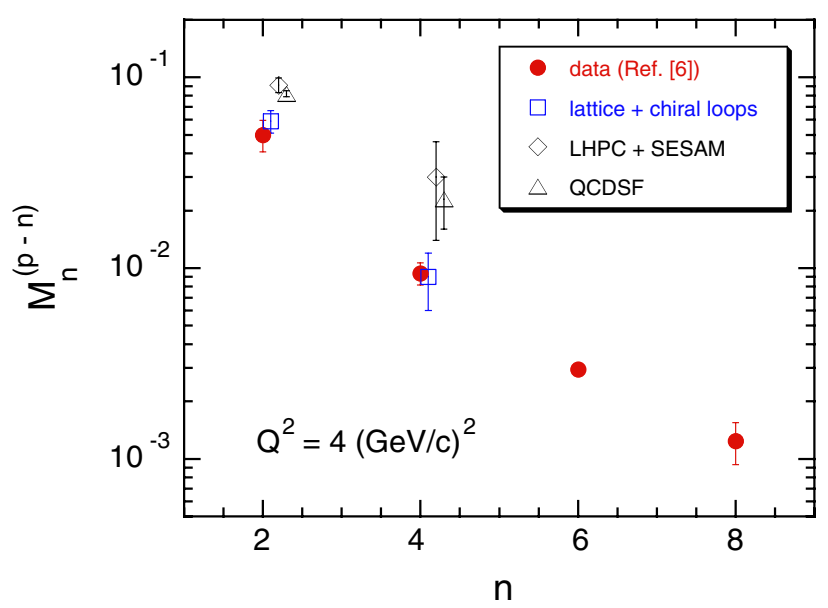

Fig. 2. Isovector $(p-n)$ moments of the nucleon $F_{2}$ structure function compared with lattice QCD simulations at $Q^{2}=$ $4(\mathrm{GeV} / c)^{2}$. Full dots are the data from ref. [6], open diamonds and triangles correspond to the lattice results from refs. [7] and [8], obtained assuming a linear extrapolation in the quark masses, and open squares are the results of ref. [9], where chiral loop effects are considered.

where $N_{n}^{(D)}$ is the moment of the function $f^{(D)}$ and $\Delta_{n}^{(o f f)}$ represents the nuclear off-shell correction. Thus, the quantity $N_{n}^{(D)} /\left[1-\Delta_{n}^{(o f f)}\right]$ is the global nuclear correction factor, i.e. the EMC effect of the deuteron in moment space. The nuclear corrections and their uncertainties have been carefully estimated in ref. [6].

The extracted neutron LT moments can be combined with the proton LT ones to form the non-singlet, isovector $(p-n)$ LT moments of the nucleon $F_{2}$ structure function. Our findings are compared against lattice QCD simulations in fig. 2. While a linear extrapolation of the lattice results to the physical pion mass overestimates our data significantly, the results obtained using the extrapolation method of ref. [9] are in much better agreement. Our results for higher moments exhibit a remarkable precision, and it would be valuable to compare them with corresponding lattice moments, because the effects of chiral loops are expected to be suppressed at large $n$.

Another interesting issue is the behavior of the neutron to proton structure function ratio $R(x) \equiv F_{2}^{n}(x) / F_{2}^{p}(x)$ in the limit $x \rightarrow 1$. This ratio can be easily related to the corresponding $d / u$ ratio of parton distributions. The "standard" value $R(x=1)=1 / 4$ is used in most of the parton distribution fits and corresponds to a vanishing $d / u$ ratio, while the value $R(x=1)=3 / 7$ is expected from pQCD arguments and corresponds to a limiting value of $1 / 5$ for the $d / u$ ratio. As shown in ref. [6] the ratio of neutron to proton moments, $M_{n}^{n} / M_{n}^{p}$, is largely independent of $Q^{2}$ and is very sensitive at large $n$ to the large- $x$ behavior of $R(x)$. Our results are shown in fig. 3 . The trend is clearly towards the "standard" value $1 / 4$ as $n$ increases, although the precision of the data does not exclude higher limiting values. From fig. 3 one can also see the impact of the nuclear corrections on the deuteron moments at large $n$ : for $n=12$, which corresponds to an average value of 


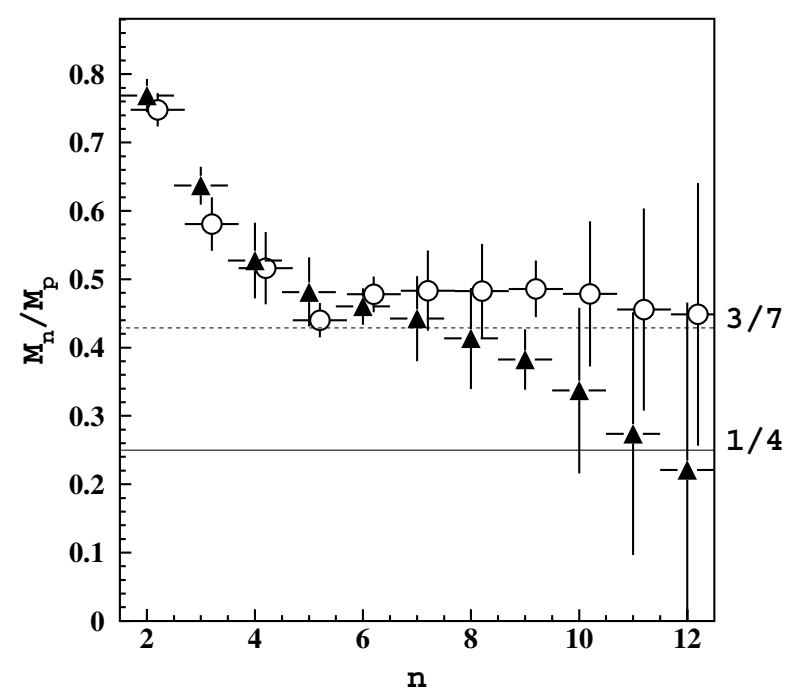

Fig. 3. Ratio of neutron to proton moments versus $n$ : full triangles and open dots corresponds to the results of ref. [6] obtained with and without the nuclear corrections, respectively. The solid (dashed) line indicates the scenario where $d / u \rightarrow 0(1 / 5)$ for $x \rightarrow 1$.

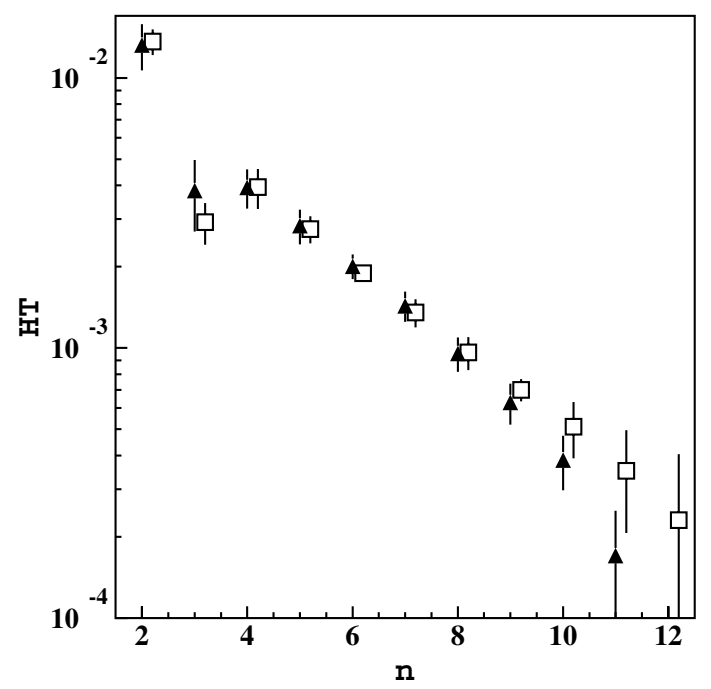

Fig. 4. Total higher twist contribution to the proton (filled triangles) and deuteron (open squares) moments evaluated at $Q^{2}=2(\mathrm{GeV} / c)^{2}$.

$x$ around 0.75 , the nuclear correction corresponds to a reduction factor of $\simeq 2$, though within large uncertainties.

Once the LT contribution to moments is determined, one can study the isospin dependence of the HT contribution. Assuming that final-state interactions and meson exchange currents have negligible impact on the HTs above $Q^{2} \simeq 1(\mathrm{GeV} / c)^{2}$, the same nuclear corrections used for the LT can be applied to the HTs. In this way the total HT contributions to proton moments and to the corrected deuteron moments can be compared, as done in fig. 4 . The comparison indicates clearly that the total HT contribution is almost independent of isospin, suggesting the possible dominance of $u d$ correlations over $u u$ and $d d$ in the nucleon [6]. Moreover, the isovector combination $(p-n)$ of the $F_{2}$ structure functions should be almost free from HT contributions within the present uncertainties.

In conclusion, we have analyzed experimental data on proton and deuteron $F_{2}$ structure functions in order to determine their moments. Using the OPE we have extracted both leading- and higher-twist contributions. By combining proton and deuteron moments we have determined the LT moments of the neutron $F_{2}$ structure function, paying particular attention to the issue of nuclear effects in the deuteron, which are increasingly important at large $n$.

The main results of our analysis can be summarized as follows:

- the ratio of neutron to proton moments is consistent with $F_{2}^{n} / F_{2}^{p} \rightarrow 1 / 4$ as $x \rightarrow 1$, although the higher value of $3 / 7$, suggested by pQCD arguments, cannot be excluded;

- the non-singlet moments are in good agreement with the lattice data $[7,8]$ only when the latter are extrapolated to physical quark masses after having taken into account chiral loops [9];

- the total contribution of HTs is found to be isospin independent, suggesting the possible dominance of $u d$ correlations over $u u$ and $d d$ in the nucleon. This implies that in the isovector combination $(p-n)$ of $F_{2}$ structure functions the HTs are almost consistent with zero within the present uncertainties.

\section{References}

1. CLAS Collaboration (M. Osipenko et al.), Phys. Rev. D 67, 092001 (2003) [arXiv:hep-ph/0301204]; M. Osipenko et al., arXiv:hep-ex/0309052.

2. CLAS Collaboration (M. Osipenko et al.), Phys. Rev. C 73, 045205 (2006) [arXiv:hep-ex/0506004]; CLAS Collaboration (M. Osipenko et al.), arXiv:hep-ex/0507098.

3. I. Niculescu, J. Arrington, R. Ent, C.E. Keppel, Phys. Rev. C 73, 045206 (2006) [arXiv:hep-ph/0509241].

4. S. Simula, Phys. Lett. B 493, 325 (2000) [arXiv:hep$\mathrm{ph} / 0005315]$.

5. S. Simula, M. Osipenko, Nucl. Phys. B 675, 289 (2003) [arXiv:hep-ph/0306260].

6. M. Osipenko, W. Melnitchouk, S. Simula, S. Kulagin, G. Ricco, Nucl. Phys. A 766, 142 (2006) [arXiv:hepph/0510189]; see also, arXiv:hep-ph/0610043.

7. LHPC Collaboration (D. Dolgov et al.), Phys. Rev. D 66, 034506 (2002) [arXiv:hep-lat/0201021].

8. QCDSF Collaboration (M. Gockeler, R. Horsley, D. Pleiter, P.E.L. Rakow, G. Schierholz), Phys. Rev. D 71, 114511 (2005) [arXiv:hep-ph/0410187].

9. W. Detmold, W. Melnitchouk, J.W. Negele, D.B. Renner, A.W. Thomas, Phys. Rev. Lett. 87, 172001 (2001) [arXiv:hep-lat/0103006]. 